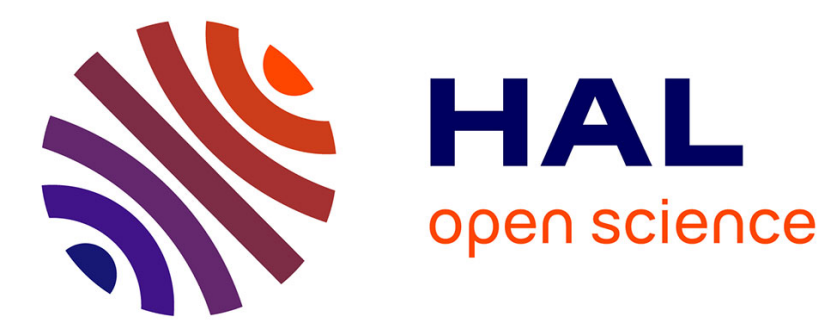

\title{
Instability of flow around a rotating, semi-infinite cylinder
}

\author{
S Derebail Muralidhar, Benoît Pier, Julian Scott
}

\section{To cite this version:}

S Derebail Muralidhar, Benoît Pier, Julian Scott. Instability of flow around a rotating, semi-infinite cylinder. Physical Review Fluids, 2016, 1, pp.053602. 10.1103/PhysRevFluids.1.053602 . hal01368877v2

\section{HAL Id: hal-01368877 \\ https://hal.science/hal-01368877v2}

Submitted on 27 Jun 2017

HAL is a multi-disciplinary open access archive for the deposit and dissemination of scientific research documents, whether they are published or not. The documents may come from teaching and research institutions in France or abroad, or from public or private research centers.
L'archive ouverte pluridisciplinaire HAL, est destinée au dépôt et à la diffusion de documents scientifiques de niveau recherche, publiés ou non, émanant des établissements d'enseignement et de recherche français ou étrangers, des laboratoires publics ou privés. 


\title{
Instability of flow around a rotating, semi-infinite cylinder
}

\author{
Srikanth Derebail Muralidhar, Benoît Pier, and Julian F. Scott \\ Laboratoire de mécanique des fluides et d'acoustique, CNRS-École centrale de Lyon- \\ Université de Lyon 1-INSA Lyon, 36 avenue Guy-de-Collongue, 69134 Écully, France
}

(Received 24 June 2016; published 16 September 2016)

\begin{abstract}
Stability of flow around a rotating, semi-infinite cylinder placed in an axial stream is investigated. Assuming large Reynolds number, the basic flow is computed numerically as described by Derebail Muralidhar et al. [Proc. R. Soc. London, Ser. A 472, 20150850 (2016)], while numerical solution of the local stability equations allows calculation of the modal growth rates and hence determination of flow stability or instability. The problem has three nondimensional parameters: the Reynolds number $\operatorname{Re}$, the rotation rate $S$, and the axial location $Z$. Small amounts of rotation are found to strongly affect flow stability. This is the result of a nearly neutral mode of the nonrotating cylinder which controls stability at small $S$. Even small rotation can produce a sufficient perturbation that the mode goes from decaying to growing, with obvious consequences for stability. Without rotation, the flow is stable below a Reynolds number of about 1060 and also beyond a threshold $Z$. With rotation, no matter how small, instability is no longer constrained by a minimum Re nor a maximum $Z$. In particular, the critical Reynolds number goes to zero as $Z \rightarrow \infty$, so the flow is always unstable at large enough axial distances from the nose. As $Z$ is increased, the flow goes from stability at small $Z$ to instability at large $Z$. If the critical Reynolds number is a monotonic decreasing function of $Z$, as it is for $S$ between about 0.0045 and 5 , there is a single boundary in $Z$, which separates the stable from the unstable part of the flow. On the other hand, when the critical Reynolds number is nonmonotonic, there can, depending on the choice of Re, be several such boundaries and flow stability switches more than once as $Z$ is increased. Detailed results showing the critical Reynolds number as a function of $Z$ for different rotation rates are given. We also obtain an asymptotic expansion of the critical Reynolds number at large $Z$ and use perturbation theory to further quantify the behavior at small $S$.
\end{abstract}

DOI: 10.1103/PhysRevFluids.1.053602

\section{INTRODUCTION}

The stability of three-dimensional boundary layers provides a rich subject of research (see Reed and Saric [1], Saric et al. [2], and references therein). Such flows are often due to rotating bodies as, for example, disks [3,4], cones [5,6], or spheres [7]. Here, we consider a semi-infinite cylinder, rotating about its axis and placed in a high-Reynolds-number axial stream, thus inducing a steady, axisymmetric, three-velocity-component boundary layer whose flow field depends on rotation and curvature of the cylinder, as we have already described in an earlier paper [8], henceforth referred to as I. In this paper, we study the stability of this flow for a wide range of parameters, to determine the effects of rotation and curvature.

As noted above, the basic flow around a rotating cylinder in an axial stream (see Fig. 1) has been extensively studied by the authors in preparation for the present stability analysis. To avoid the flow separation typically induced by sharp corners, a smooth nose is assumed at the front of the cylinder. The problem has two nondimensional control parameters: a Reynolds number $\operatorname{Re}=U_{\infty} a / v$, constructed using the incident velocity $U_{\infty}$ and cylinder radius $a$, and a rotation rate $S=\Omega a / U_{\infty}$, where $\Omega$ is the angular velocity of the cylinder. Assuming large Reynolds number, the basic flow comprises an axisymmetric boundary layer. Initially thin compared to the cylinder radius, the boundary-layer thickness increases with axial distance, becoming comparable to the cylinder radius at large axial distances of order $a$ Re. The thickness nonetheless remains small compared with the downstream distance, leading to a separation of length scales (asymptotic in 


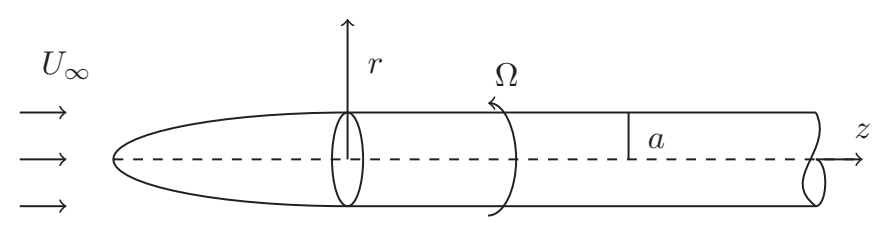

FIG. 1. Schematic diagram of the problem.

the assumed large Reynolds number) between the axial and radial directions and allowing use of a boundary-layer approximation. However, once the thickness is comparable to $a$, effects of surface curvature and the centrifugal force due to rotation become important and must be included in the boundary-layer equations. The intervention of curvature and rotation ax axial distances of order $a \mathrm{Re}$ is the reason why we focus on this region. Although the basic flow depends on the nose geometry at streamwise distances of order $a$, boundary-layer development forgets such details and the flow becomes independent of the nose profile at larger distances, in particular those of order $a$ Re. Interested readers should refer to I for more details of the basic flow, including the appearance of a wall jet for $S$ above about 4 and asymptotic analyses of the limits of large rotation rate and large streamwise distance.

Stability of the Blasius boundary layer on a flat plate has been extensively studied, the primary instability being due to growth of Tollmien-Schlichting waves (see, for example, [9-11]). Stability analysis of a nonrotating cylinder placed in an axial stream was conducted by Tutty [12]. They found that nonaxisymmetric modes have a lower critical Reynolds number than axisymmetric modes and that the flow is stable at all streamwise distances for Reynolds numbers below 1060. Above this critical value, the flow is unstable only for a range of streamwise distance and reverts to being stable at sufficiently large distances. Vinod and Govindarajan [13] studied the secondary instability and also showed that the flow is stable according to inviscid theory.

There have been few studies of the stability of a rotating cylinder placed in an axial stream. Kao and Chow [14] appear to be the first to consider the rotating case, but their basic flow is erroneous because they do not include the centrifugal term in the radial momentum equation. They also studied the nonrotating case, but the results are in disagreement with [12]. This disagreement was noted by Herrada et al. [15], who were the first to formulate the correct basic-flow equations. Results were obtained for a range of rotation rates between 0.1 and 1 , and are in excellent agreement with our results. They observed that the critical Reynolds numbers were much lower than for the nonrotating case. This motivated us to study the rotating case in a more systematic manner, in particular the range of small rotation rates. To our surprise, we found that the stability characteristics, in particular the critical curves, were significantly dependent on the rotation rate for values of $S$ much lower than the ones covered in Ref. [15]. The elucidation of these differences between the nonrotating case and the rotating case at very low $S$ is one of the main topics of this paper.

To our knowledge, the only published experimental work on a rotating cylinder which matches the geometry of the present study is by Kegelman et al. [16]. They give visualizations of different instability modes developing on the cylinder. However, the cylindrical section in their experiments is not long enough to reach distances at which the flow forgets the details of the nose profile (let alone to reach distances at which curvature and rotation become important for the basic flow). Thus, quantitative comparison between our results and [16] is inappropriate and is not attempted.

Formally, the basic-flow and stability equations for the rotating cylinder are the same as those of a vortex core with axial flow (in the quasicylindrical approximation, see [17]). However, the two flows are quite different and it would be rash to draw parallels in terms of stability. In particular, vortex breakdown is associated with an adverse axial pressure gradient of the basic flow, whereas the rotating cylinder has a favorable pressure gradient.

The local stability equations are derived in Sec. II. The numerical scheme is described in Sec. III, along with results of validation. Section IV contains the main results. In Sec. IV C, the limit of low rotation rate is examined in more detail and quantified using a perturbation method. 


\section{PROBLEM FORMULATION}

A semi-infinite cylinder of radius $a$, which rotates about its axis at angular velocity $\Omega$, is placed in an axial stream of velocity $U_{\infty}$ (see Fig. 1), the fluid being incompressible and of viscosity $v$ and density $\rho$. Lengths, times, velocities, and pressures are nondimensionalized using $a, a / U_{\infty}, U_{\infty}$, and $\rho U_{\infty}^{2}$. Adopting cylindrical coordinates $(z, r, \theta)$, the basic (steady and axisymmetric) flow has velocity components $U_{z}, U_{r}, U_{\theta}$ and associated pressure denoted by $P$. As noted in the Introduction, there are two control parameters, namely, the Reynolds number Re and the rotation rate $S$, given by

$$
\begin{aligned}
\operatorname{Re} & =\frac{U_{\infty} a}{v}, \\
S & =\frac{\Omega a}{U_{\infty}} .
\end{aligned}
$$

To avoid possible confusion, we remark that $U_{z}, U_{r}, U_{\theta}$, and $P$ were denoted by $u_{z}, u_{r}, u_{\theta}$, and $p$ in I, whereas these lower-case quantities are used to represent the flow perturbation in this paper.

We assume large Reynolds number from here on and restrict attention to the flow over the constantradius part of the cylinder $z>0$. As discussed in the Introduction, large Reynolds number leads to a separation of length scales in which streamwise evolution of the basic flow is slow compared with its radial variation. Not only does this allow use of the boundary-layer approximation to describe the basic flow, but it is also a prerequisite for local stability analysis. Boundary-layer theory implies that $U_{r}$ is small compared with $U_{z}$ and $U_{\theta}$. Neglect of $U_{r}$ is the second ingredient of local stability theory.

Under these assumptions, the steady and axisymmetric base-flow components are governed by a generalization of the classical Prandtl boundary-layer equations that allow for the additional effects of both curvature and rotation. Near the nose, the boundary layer is thin compared with the cylinder radius. Thus, in that region, curvature effects are negligible and the flow is close to the Blasius profile of a flat plate. Using a Blasius inlet condition, the entire base flow is then obtained by integrating the boundary-layer equations along the $z$ direction. See I for full theoretical and numerical details about the basic flow.

The linear stability equations are obtained in the usual manner: by adding infinitesimal perturbations $\left(u_{z}, u_{r}, u_{\theta}, p\right)$ to the basic-flow quantities $\left(U_{z}, U_{r}, U_{\theta}, P\right)$, using the nondimensionalized Navier-Stokes equations, and neglecting terms which are nonlinear in the perturbations. The local approximation is then adopted: $U_{r}$ is neglected, as are variations of $U_{z}$ and $U_{\theta}$ with respect to $z$. Invariance with respect to $z, \theta$, and $t$ allows modal solutions of the form

$$
\left(\begin{array}{l}
u_{z} \\
u_{r} \\
u_{\theta} \\
p
\end{array}\right)=\left(\begin{array}{l}
v_{z}(r) \\
v_{r}(r) \\
v_{\theta}(r) \\
q(r)
\end{array}\right) e^{i(\alpha z+m \theta-\omega t)}
$$

Here, $\omega$ is the complex frequency, while $\alpha$ and $m$ are the axial and azimuthal wave numbers, which are, respectively, real and integer modal parameters. Note that modes occur in complex-conjugate pairs, related by $\alpha \leftrightarrow-\alpha, m \leftrightarrow-m$, and $\omega \leftrightarrow-\omega^{*}$, where $*$ denotes complex conjugation. It is this conjugation symmetry which allows the construction of physically meaningful (i.e., real) solutions by combining a mode and its conjugate. Conjugation symmetry allows us to restrict attention to $\alpha \geqslant 0$, while $m$ can take any integer value.

Substituting the modal form for the perturbation in the local stability equations gives

$$
\begin{aligned}
i \alpha v_{z}+v_{r}^{\prime}+\frac{1}{r} v_{r}+\frac{i m}{r} v_{\theta} & =0 \\
i\left(\alpha U_{z}+\frac{m U_{\theta}}{r}-\omega\right) v_{z}+U_{z}^{\prime} v_{r}+i \alpha q & =\frac{1}{\operatorname{Re}}\left[v_{z}^{\prime \prime}+\frac{1}{r} v_{z}^{\prime}-\left(\alpha^{2}+\frac{m^{2}}{r^{2}}\right) v_{z}\right],
\end{aligned}
$$




$$
\begin{aligned}
i\left(\alpha U_{z}+\frac{m U_{\theta}}{r}-\omega\right) v_{r}-\frac{2 U_{\theta}}{r} v_{\theta}+q^{\prime} & =\frac{1}{\operatorname{Re}}\left[v_{r}^{\prime \prime}+\frac{1}{r} v_{r}^{\prime}-\left(\alpha^{2}+\frac{m^{2}+1}{r^{2}}\right) v_{r}-\frac{2 i m}{r^{2}} v_{\theta}\right], \\
i\left(\alpha U_{z}+\frac{m U_{\theta}}{r}-\omega\right) v_{\theta}+\left(U_{\theta}^{\prime}+\frac{U_{\theta}}{r}\right) v_{r}+\frac{i m}{r} q & =\frac{1}{\operatorname{Re}}\left[v_{\theta}^{\prime \prime}+\frac{1}{r} v_{\theta}^{\prime}-\left(\alpha^{2}+\frac{m^{2}+1}{r^{2}}\right) v_{\theta}+\frac{2 i m}{r^{2}} v_{r}\right],
\end{aligned}
$$

where the primes denote derivatives with respect to $r$. The boundary conditions are

$$
\begin{gathered}
v_{z}(1)=v_{r}(1)=v_{\theta}(1)=0, \\
v_{z}(\infty)=v_{r}(\infty)=v_{\theta}(\infty)=0 .
\end{gathered}
$$

The modes are determined as nonzero solutions of the above boundary-value problem. The problem contains the basic-flow velocity profiles $U_{z}(r)$ and $U_{\theta}(r)$, which are determined by solving the boundary-layer equations, as described in I. As noted in the Introduction, the basic flow becomes independent of the nose geometry for streamwise distances much greater than $a$, i.e., large $z$, and we focus on this region. As shown in I, $U_{z}(r)$ and $U_{\theta}(r)$ then depend only on the parameters $Z=z \operatorname{Re}$ and $S$, of which the former is a version of streamwise distance, scaled such that the boundary-layer thickness is of order $a$ when $Z$ is of order 1 . Thus, the physical parameters of the problem are Re, $Z$, and $S$, while it also contains the modal parameters $\alpha$ and $m$. The set of differential equations and boundary conditions given above form an eigenvalue problem for determination of possible values of the complex frequency $\omega=\omega_{r}+i \omega_{i}$, whose imaginary part gives the modal growth rate. Expressing the eigenvalues in terms of the other parameters of the problem yields the dispersion relation

$$
\omega=F(\alpha, m, \operatorname{Re}, Z, S) .
$$

Numerical solution of the eigenvalue problem is described in the next section. The physical parameters Re, $Z$, and $S$ and modal parameters $\alpha$ and $m$ are then varied, looking for growing modes, i.e., eigenvalues with $\omega_{i}>0$, which are symptomatic of local instability.

\section{NUMERICAL METHOD}

Prior to numerical discretization, we transform the radial coordinate as in I. Because the boundarylayer thickness goes to zero like $Z^{1 / 2}$ at small $Z$, the radial coordinate is first replaced by $\sigma=$ $(r-1) /(2 Z)^{1 / 2}$ to improve the radial resolution in that limit. The semi-infinite range of $\sigma$ is then transformed to a finite interval using

$$
x=\frac{\sigma-\hat{\sigma}}{\sigma+\hat{\sigma}}, \quad \sigma \in[0, \infty] \rightarrow x \in[-1,1]
$$

where $\hat{\sigma}>0$ is a numerical parameter allowing some control over the distribution of the discrete points introduced below.

Also as in I, rewriting the local stability equations using the coordinate $x$, Chebyshev collocation is used to discretize the problem. We introduce the $N$ collocation points

$$
x_{n}=\cos \left(\frac{n \pi}{N-1}\right), \quad 0 \leqslant n<N
$$

where, to avoid interpolation, $N$ has the same value as in the calculation of the basic flow. Note that, according to (11), $\hat{\sigma}$ divides the flow into two ranges, $\sigma<\hat{\sigma}$ and $\sigma>\hat{\sigma}$, containing equal numbers of points. The smaller $\hat{\sigma}$, the better the resolution near the cylinder and the worse the resolution at large $\sigma$, the opposite being true if $\hat{\sigma}$ is increased. The variables $v_{z}, v_{r}$, and $v_{\theta}$ are represented by their values at all collocation points, whereas only the values of $q$ for $0<n<N-1$ are used. As is usual in Chebyshev collocation, derivatives are expressed using polynomial fitting: $(N-1)$ th degree polynomials are fitted to the discrete values of $v_{z}, v_{r}$, and $v_{\theta}$ and an $(N-3)$ th one to those of $q$. This allows approximation of derivatives as matrices obtained by differentiating the polynomials. Equations (4)-(7) are applied at $x_{n}$ for $0<n<N-1$, giving $4 N-8$ equations for the $4 N-2$ 
(a)

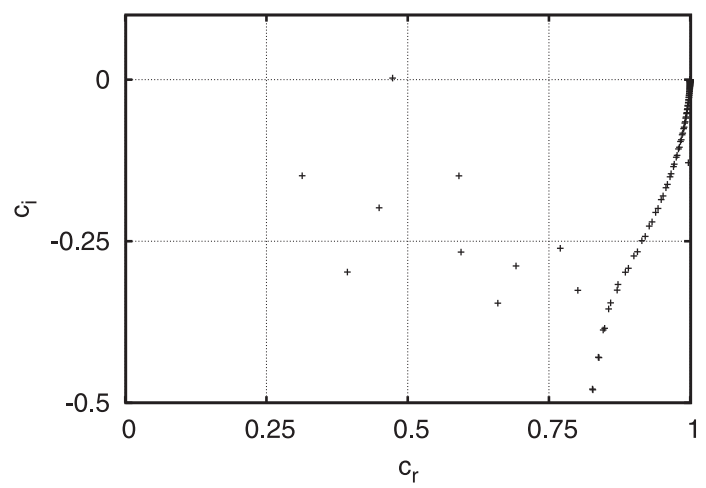

(b)

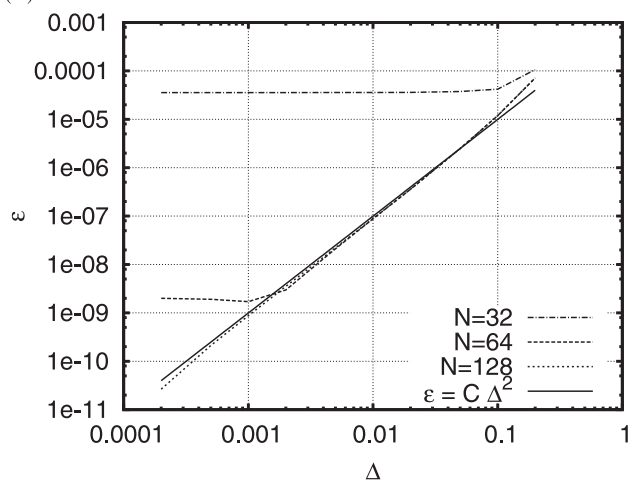

FIG. 2. (a) Eigenspectrum in the complex $c$ plane for $\operatorname{Re}=2000, \alpha=0.2, Z=0.5, m=1$, and $S=0.01$. (b) Relative error plot as a function of the axial discretization of the basic flow for different values of $N$.

discrete values of $v_{z}, v_{r}, v_{\theta}$, and $q . q$ is eliminated as described in Appendix C1 of [18], as are the boundary values of $v_{z}, v_{r}$, and $v_{\theta}$ using (8) and (9). The result is a $(3 N-6) \times(3 N-6)$ standard matrix eigenvalue problem with eigenvalue $\omega$ and eigenvector whose elements are the discrete values of $v_{z}, v_{r}$, and $v_{\theta}$ at $x=x_{n}$ for $0<n<N-1$.

Figure 2(a) shows an example of a numerically computed eigenspectrum in the complex phase velocity $\left(c=c_{r}+i c_{i}\right)$ plane (where $\left.c=\omega / \alpha\right)$. It consists of a discretized version of the continuous spectrum (which arises because the flow domain is semi-infinite in the radial direction) and a set of discrete eigenvalues, one of which has $c_{i}>0$ and therefore represents a growing mode. We conclude that the flow is unstable for the given values of $\operatorname{Re}, Z$, and $S$. The continuous spectrum can be safely neglected as it always lies in $c_{i} \leqslant 0$, originating at $c=1-i \alpha / \operatorname{Re}$ and extending downwards in the complex $c$ plane (see the asymptotic analysis in Ref. [19]).

The code was tested by observing the sensitivity of the most unstable (largest $c_{i}$ ) discrete eigenvalue (which is the one of principal interest) to changes in the numerical parameters $N, \hat{\sigma}$, and $\Delta$, where $\Delta$ is the axial step used in the basic-flow computation [recall from I that the basic flow was obtained by integration of the boundary-layer equations using small steps $\Delta$ in $\zeta=(2 Z)^{1 / 2}$ ]. Figure 2(b) shows a log-log plot of the relative error $\epsilon$ of the computed eigenvalue with $\hat{\sigma}=5$ and the parameters given in Fig. 2(b) as a function of $\Delta$ for different values of $N$. The error is computed by comparison with the case $N=128$ and $\Delta=0.0001$. It will be seen that, for $N=64$ and 128 , the accuracy is mainly limited by axial discretization. The results are consistent with the use of a scheme which is second-order accurate in $\Delta$ to compute the basic flow, as can be seen by comparison with the reference line on the plot. The mapping parameter $\hat{\sigma}$ was found to affect the precision at only the 10 th decimal place when varied between $2-6$. After studying such convergence results for numerous sets of parameters, we decided to use $N=64, \hat{\sigma}=5$, and $\Delta=0.001$ for the computations. This gives better than eight decimal places of accuracy. Note that, even though the continuous spectrum is not well resolved, this does not significantly affect the accuracy with which the most unstable eigenvalue is computed. The numerical eigenfunctions were also validated by comparing their exponential decay rate at large radial distances with the asymptotic decay rate given by the streamwise wave number $\alpha$ (see [19]). Good agreement was found.

For given $m$ and $S$, the neutral curve is defined as the boundary of the region in the Re- $Z$ plane for which a growing mode exists. Another way of putting this is that it is the curve of zero maximum growth rate, with the maximum taken over the discrete spectrum and all $\alpha$. A first approximation to the neutral curves was obtained by plotting the contour of zero maximum growth rate in the Re- $Z$ plane using a rectangular grid of values. This was carried out for different values of $m$ and $S$. However, obtaining accurate neutral curves with such a method requires a very fine grid, making it computationally expensive. A faster, multivariate Newton-Raphson scheme was developed to obtain 


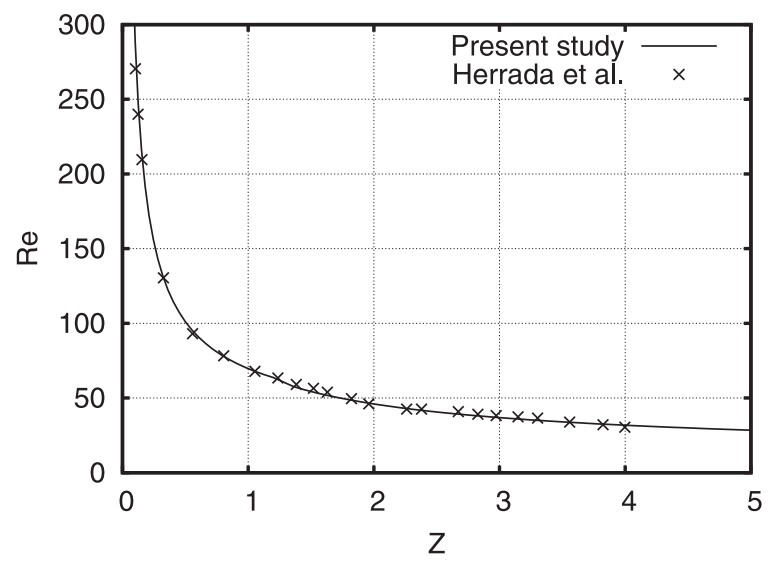

FIG. 3. Comparison of the neutral curve for $m=1, S=0.1$ with results of Herrada et al. [15].

more precise results with values taken from the contour plot to initialize the iteration. The neutral point $\alpha, \operatorname{Re}$ at a given $m, Z, S$ is obtained by simultaneously solving the system of equations

$$
\begin{aligned}
\omega_{i}(\alpha, \mathrm{Re}) & =0, \\
\frac{\partial \omega_{i}}{\partial \alpha}(\alpha, \mathrm{Re}) & =0 .
\end{aligned}
$$

The Newton-Raphson scheme requires computation of derivatives of $\omega_{i}$ with respect to $\alpha$ and $\mathrm{Re}$, which were obtained using centered finite differencing. The solution was considered to have converged when the norm of the residuals was less than $10^{-7}$. The result was used to initialize the iteration at the next step in $Z$, and the process continued for a range of values of $Z$.

The code was validated by comparing the results for the neutral curve with $S=0.1$ and $m=1$ with those of [15]. Figure 3 shows good agreement. Since the results of [15] for the nonrotating case are known to be in agreement with those of [12], a comparison of our results with [12] will not be presented, although we did check they agreed.

\section{RESULTS}

\section{A. Eigenspectra and growth-rate plots}

The eigenspectra for the nonrotating case are qualitatively similar to that of the Blasius boundary layer, there being one distinguished eigenvalue close to the real axis in the complex $c$ plane. This eigenvalue may lie in either $c_{i}>0$ or $c_{i} \leqslant 0$, the remainder of the discrete spectrum being in $c_{i}<0$ and well separated from the real $c$ axis. Similarity with the Blasius flow might be expected because the basic flow without rotation has a velocity profile $U_{z}(r)$, whose form resembles the Blasius profile, which it approaches in the limit $Z \rightarrow 0$. It is the sign of $c_{i}$ for the distinguished mode which controls flow stability in the nonrotating case, hence also for sufficiently small values of $S$.

Figure 2(a) shows an example with small rotation rate $(S=0.01)$ : the eigenspectrum is essentially the same as for the nonrotating case at the same values of $\operatorname{Re}, \alpha, Z$, and $m$. However, because it is close to the real $c$ axis, the slight perturbation of the distinguished mode at small $S$ may suffice for $c_{i}$ to change sign, with obvious consequences for flow stability. This does, in fact, happen for the values of $\operatorname{Re}, \alpha, Z$, and $m$ used in Fig. 2(a): $c_{i}<0$ when $S=0$ and $c_{i}>0$ when $S=0.01$. This is a reflection of a surprising sensitivity of flow stability to small amounts of rotation, an important theme of this paper which will be further elucidated by later results.

As $S$ is increased to larger values, the eigenspectrum changes character and one can no longer think in terms of a single distinguished mode near the real $c$ axis which controls stability. Figure 4(a) 
(a)

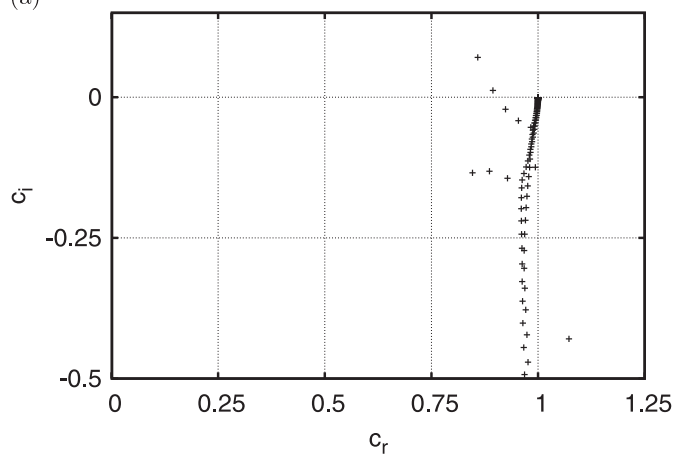

(b)

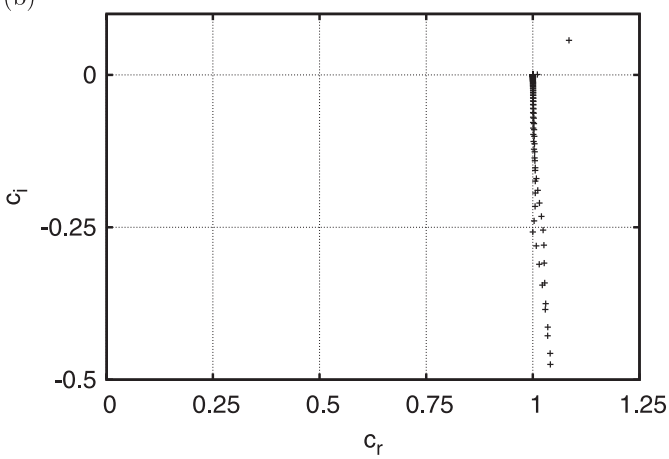

FIG. 4. Eigenspectra for (a) $\operatorname{Re}=200, \alpha=0.16, Z=12.5, m=3$, and $S=0.2$, (b) $\operatorname{Re}=200, \alpha=0.04$, $Z=12.5, m=1$, and $S=0.5$.

shows an example in which two discrete modes are unstable. Thus, for the rotating case, there can be more than one growing mode. When this occurs, the mode with the largest $c_{i}$ is more important because it grows fastest according to the linear theory used here. Figure 4(b) gives an example in which there is a growing mode with $c_{r}>1$, despite the fact that the maximum value of $U_{z}(r)$ is 1. Howard and Gupta [20] have shown that a modified semicircle theorem (which places limits on $\left.c_{r}\right)$ can be obtained for general swirling flows, but only for axisymmetric disturbances $(m=0)$ in the inviscid case. We are unaware of any such result for nonaxisymmetric disturbances. The given example shows that the phase velocity of a mode can exceed the maximum of $U_{z}(r)$ when $S>0$.

From here on, we focus on the fastest growing discrete mode. Overall instability or stability of the flow is determined by the sign of the maximum growth rate $\omega_{i}^{\max }$, the maximum being taken over all $m$ and $\alpha$. However, before taking this step, it is perhaps interesting to consider the behavior of the growth rate $\omega_{i}$ as a function of $m$ and $\alpha$. We never found a case in which $\omega_{i}^{\max }>0$ arose from $m \leqslant 0$ modes, so we mostly restrict attention to $m>0$ in what follows.

Figure 5(a) shows an example for which $\omega_{i}$ is plotted as a function of $\alpha$ for different values of $m$. We see that the $m$ which yields the largest $\omega_{i}$ depends on $\alpha$ and that the overall maximum $\omega_{i}^{\max }$ arises from $m=1$ (it is given by the peak in the $m=1$ curve). This is not always the case, as is apparent from Fig. 5(b), for which $\omega_{i}^{\max }$ arises from $m=2$. In the absence of rotation, we found that $\omega_{i}^{\max }$ was always associated with either $m=1$ or 2 when the flow was unstable, in agreement with the results of [12] for the particular case $\operatorname{Re}=15000$. However, higher values of $m$ can arise for nonzero $S$ and we found no general rule concerning the $m$ associated with $\omega_{i}^{\max }$ in the presence of rotation. We remark that, for the quite different case of flow in a rotating pipe, Pedley [21] also found that the $m$ yielding the maximum overall growth rate depends on the physical parameters of the flow.

Negative values of $m$ were also examined. In the nonrotating case, reflection symmetry with respect to any plane containing the cylinder axis implies that the growth rates for $m$ are the same as for $-m$. For nonzero $S$, we found that negative $m$ yields lower growth rates than positive $m$, as illustrated by Fig. 5(c). Attention is restricted to $m>0$ from here on.

Figure 5(d) shows cases for which $\omega_{i}(\alpha)$ has more than one local maximum. As the physical parameters are varied, the overall maximum can jump discontinuously from one local maximum to another, a phenomenon encountered later. The effect of such a jump is apparent in Fig. 6(c) of [15] and in results of the next subsection.

\section{B. Overall maximum growth rate, neutral and critical curves}

Contour plots of overall maximum growth rate $\omega_{i}^{\max }$ in the Re- $Z$ plane are shown for three values of $S$ in Fig. 6. The contours $\omega_{i}^{\max }=0$ are the critical curves, which form the boundary between flow stability and instability. (Note that we use the term neutral curve for the stability boundary of 
(a)
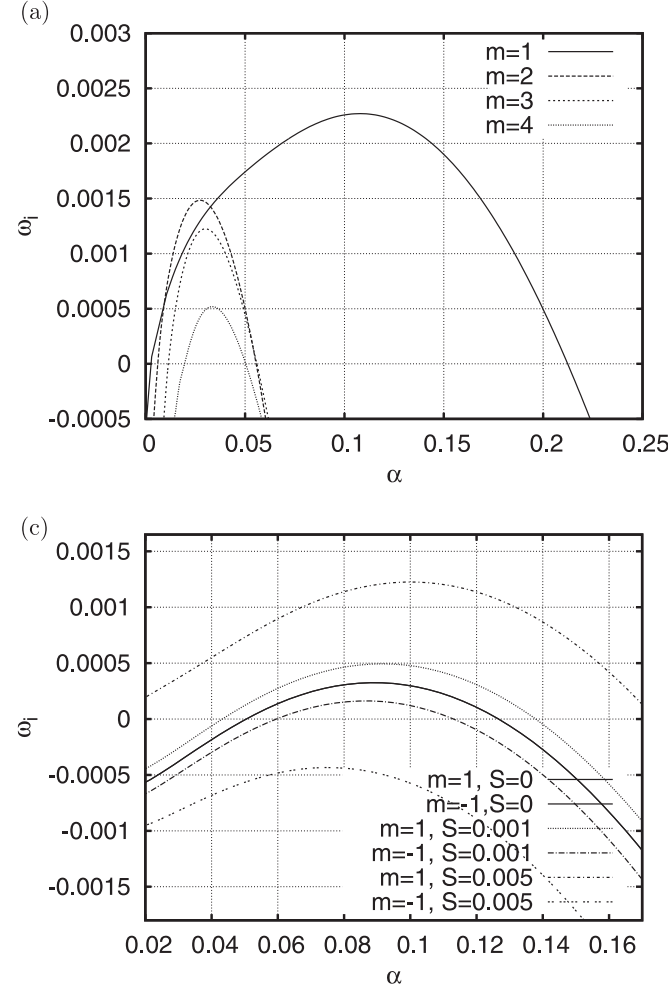

(b)
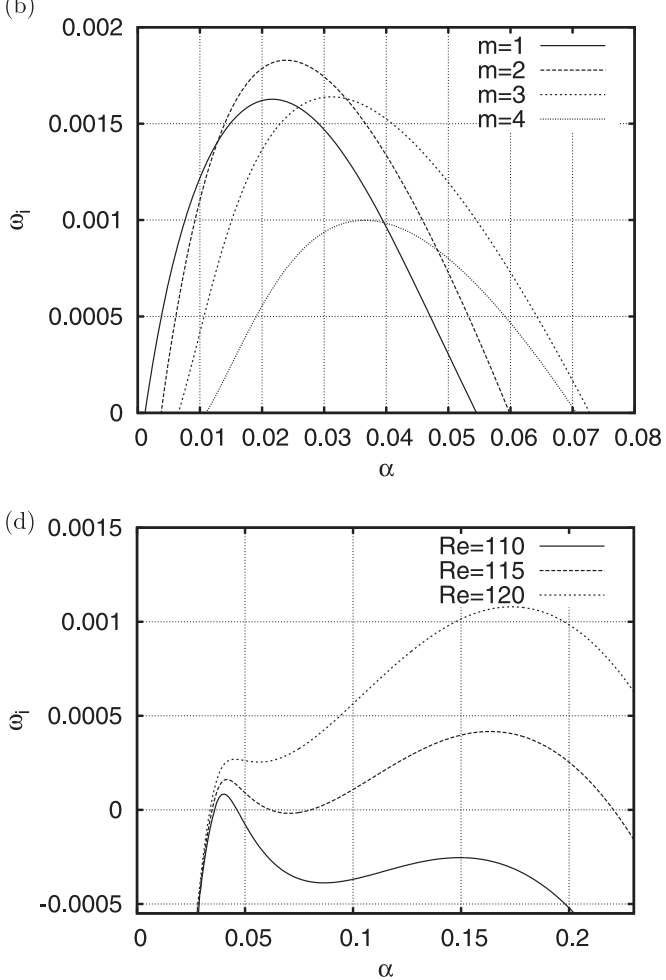

FIG. 5. Plots of temporal growth rate as a function of $\alpha$ : (a) $\operatorname{Re}=2000, Z=0.5$, and $S=0.01$, (b) $\operatorname{Re}=2000, Z=2$, and $S=0.01$, (c) $\operatorname{Re}=2000, Z=0.5$ for $m=1$ and -1 and different (small) values of $S$, (d) $Z=0.5, S=0.1, m=1$ for different values of Re.

a single value of $m$, while a critical curve allows for all $m$.) Figure 6(a) shows that the nonrotating flow is always stable above a certain value of $Z(0.8001)$, i.e., at sufficiently large downstream distances, and also below a certain value of $\operatorname{Re}(1059.5$, in accord with [12]). The other two cases are quite different: instability has neither an upper limit in $Z$ nor a lower limit in Re. Note the small growth rates in the unstable region for all three cases. This reflects control of small- $S$ stability by a distinguished mode close to the real $c$ axis, as does Fig. 7. Note also the large differences between the contour plots, a result which is perhaps at first surprising, but is, in fact, a logical consequence of a controlling mode close to the real $c$ axis for $S=0$.

As noted towards the end of Sec. III, obtaining accurate neutral curves using contour plots is computationally expensive compared to Newton-Raphson iteration and varying $Z$ in small steps, and the same is even more true for critical curves because different $m$ must be accounted for. For this reason, we use Newton-Raphson iteration (as described in Sec. III) to follow the neutral curves, then combine the results for different $m$ to obtain the critical curve. This is achieved by minimization of the neutral Reynolds number over $m$ for given $Z$ and $S$.

Figure 8(a) shows neutral curves for the first three nonaxisymmetric modes and $S=0.1$. The critical Reynolds number arises from the $m=1$ mode for $Z>1.3$ and from $m=2$ for $Z$ between $0.05-1.3$. Higher $m$ take over for $Z$ less than about 0.05 . These do not contribute to the plots of Fig. 8(a) because the critical Reynolds number lies above the range shown. As noted earlier, $m=1$ and 2 control stability in the nonrotating case and here we see that they are the most important modes for small $S$ as well. Indeed, we found that, when $Z>0.1$, criticality was associated with $m=1$ or 2 in all cases studied. Figure 8(b) shows plots of the axial wave number corresponding to neutrality. The jumps are a consequence of the existence of two local maxima in $\omega_{i}(\alpha)$, a scenario discussed 

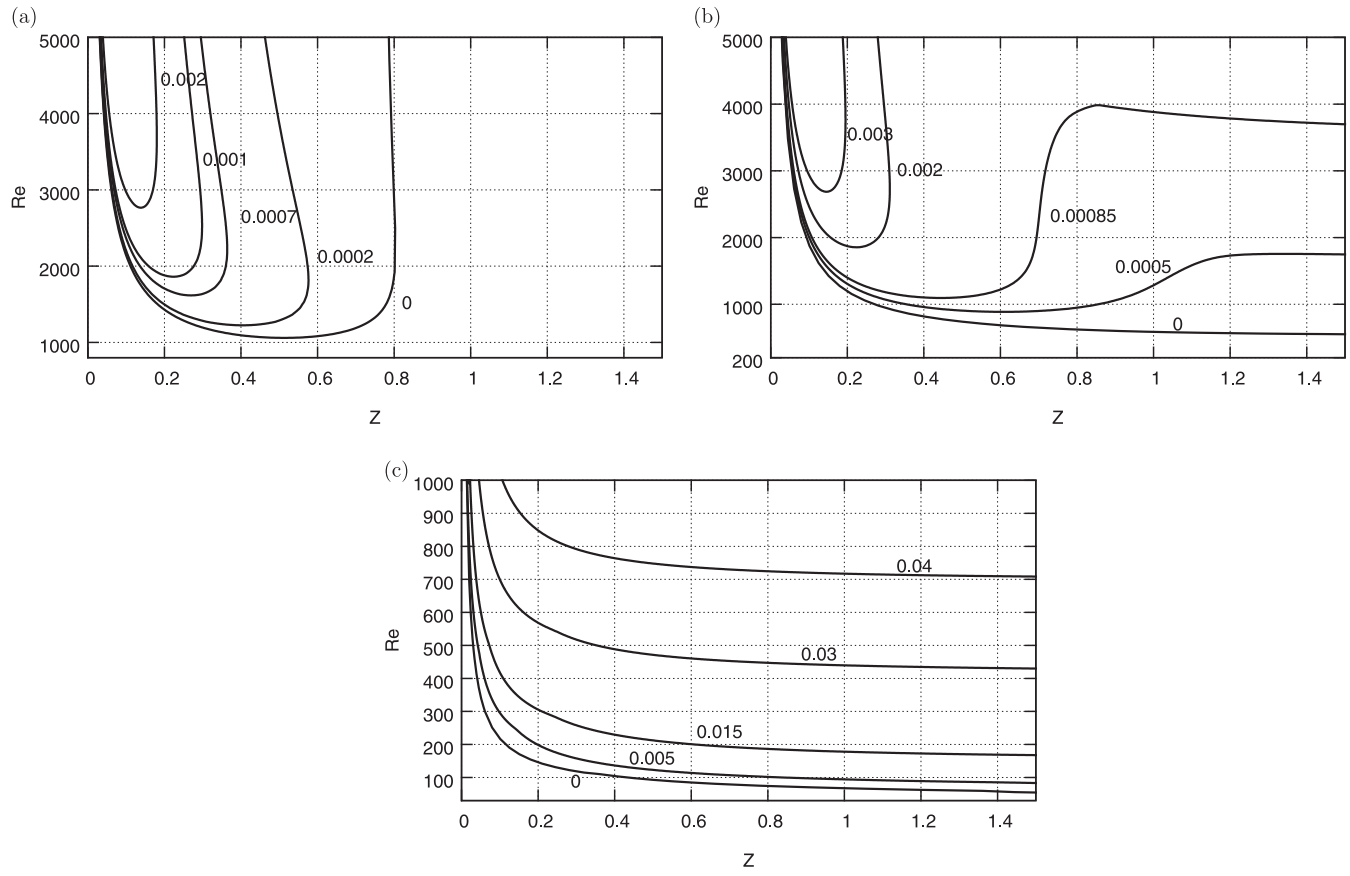

FIG. 6. Contour plots of $\omega_{i}^{\max }$ for (a) $S=0$, (b) $S=0.005$, and (c) $S=0.1$.

earlier and illustrated by Fig. 5(d). As $Z$ is varied, neutrality is controlled first by one of the maxima, then by the other. At the jump, both maxima give $\omega_{i}=0$.

Figure 9 shows critical curves and corresponding wave-number plots for values of $S$ between $0-0.012$. The sensitivity of flow stability to small amounts of rotation is again apparent. As noted earlier, when $S=0$ there is a minimal Reynolds number and a maximal $Z$ for instability, neither of which persist to the rotating case. A given value of the Reynolds number corresponds to a horizontal line in Fig. 9(a), whose intersections with the critical curve yield boundaries in $Z$ separating regions of stability and instability. With or without rotation, the flow is stable at sufficiently small $Z$, but each time the critical curve is crossed, the flow changes stability. In the nonrotating case, stability at small $Z$ can either persist to all $Z$ (if $\operatorname{Re} \leqslant 1059.5$ ) or there is a range of instability, beyond which the flow is again stable. The rotating case is quite different because the critical Reynolds number
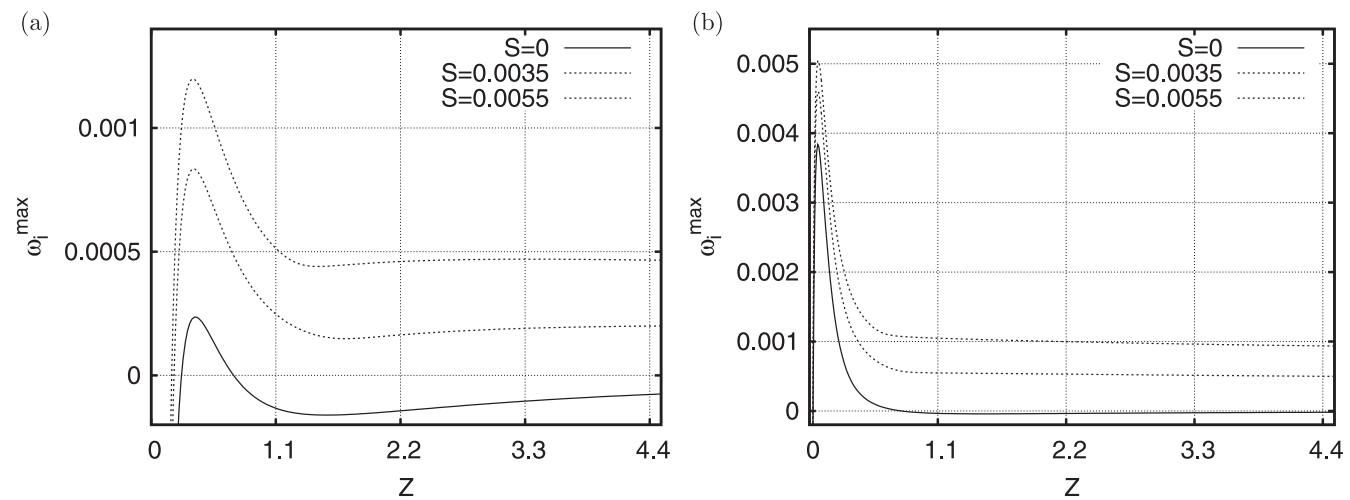

FIG. 7. Plots of $\omega_{i}^{\max }$ as a function of $Z$ for different (small) values of $S$ and (a) $\operatorname{Re}=1250$, (b) $\operatorname{Re}=5000$. 

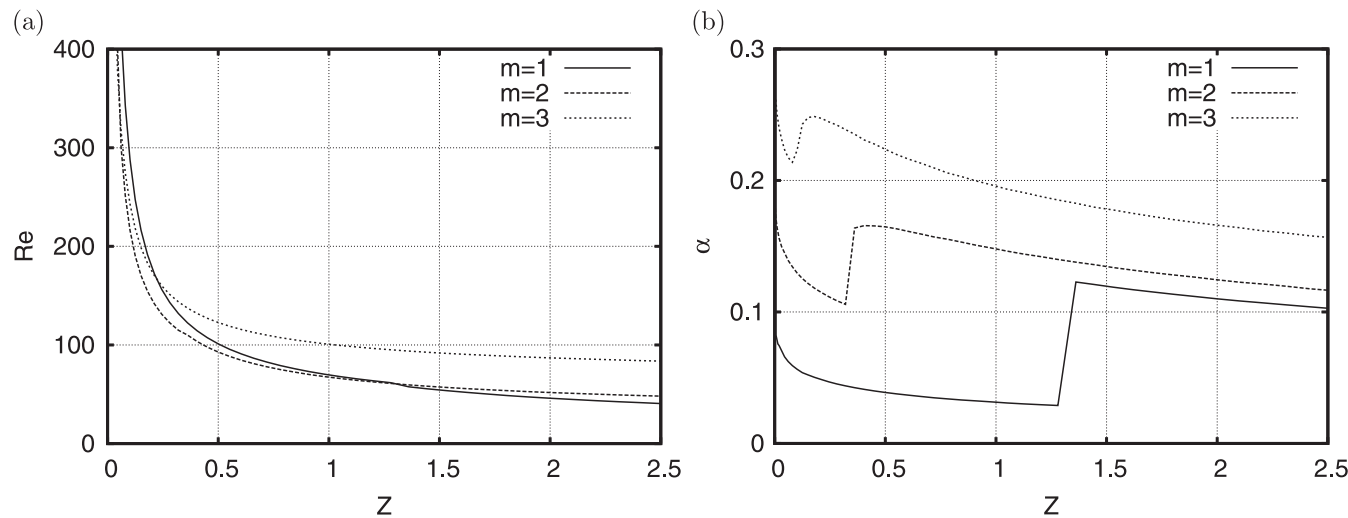

FIG. 8. (a) Neutral curves and (b) corresponding wave-number plots for $m=1,2$, and 3 and $S=0.1$.

decreases to zero at large $Z$. As a result, the flow is unstable for sufficiently large $Z$. If the critical Reynolds number is a monotonically decreasing function of $Z$, as it is for small $S$ above a certain threshold value equal to about 0.0045 , there is a single intersection of the critical curve with any given line of constant Re, resulting in stability at small $Z$, followed by instability once the critical curve is crossed. On the other hand, the critical curve is nonmonotonic for $S$ below the threshold, as illustrated by Fig. 9(a) for three such values of $S>0$. For values of Re between the local minimum and maximum of the curve, there are then three stability boundaries, hence, four distinct regions in $Z$ : stable, unstable, stable, unstable as $Z$ increases. Note that increasing $S$ lowers the critical Reynolds number, i.e., rotation has a destabilizing effect.

Figure 10 concerns $S$ between 0.1 and 1 . According to Fig. 10(a), the critical Reynolds number remains a decreasing function of $Z$ and $S$, while Fig. 10(b) shows jumps in the critical wave number. Some of these jumps were identified as between local maxima of $\omega_{i}(\alpha)$ for a single $m$, while others correspond to jumps between different $m$.

Figure 11 gives results for higher values of $S$. Close inspection of Fig. 11(a) reveals that the critical Re is no longer a monotonic decreasing function of $Z$ for $S=10$ and 15 . This is more apparent in Fig. 12, which extends the range of $Z$ to much higher values using a logarithmic scale. For $S$ exceeding a threshold value of about 5 , the critical curve is nonmonotonic, as it is for $S$ below the small- $S$ threshold of about 0.0045 . This leads to the same qualitative conclusion concerning stability, namely, the possibility, depending on Re, of more than one stability or instability boundary
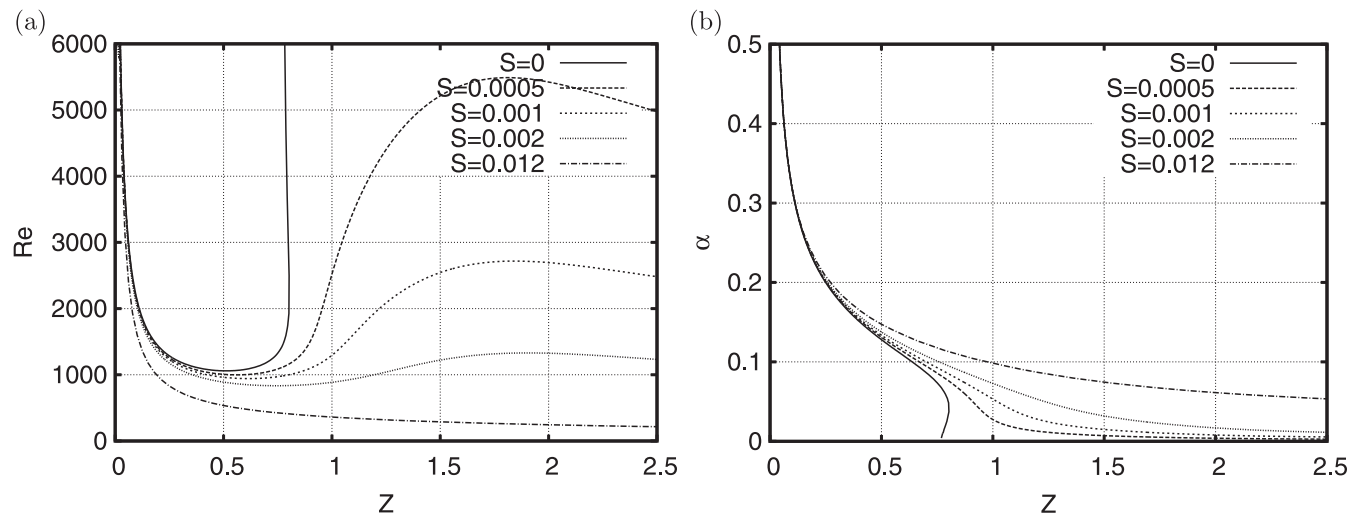

FIG. 9. (a) Critical curves and (b) corresponding wavenumber plots for values of $S$ between 0 and 0.012 . 
(a)

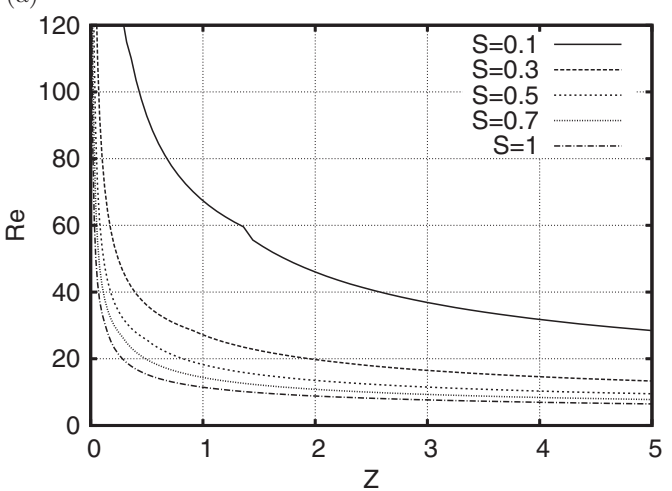

(b)

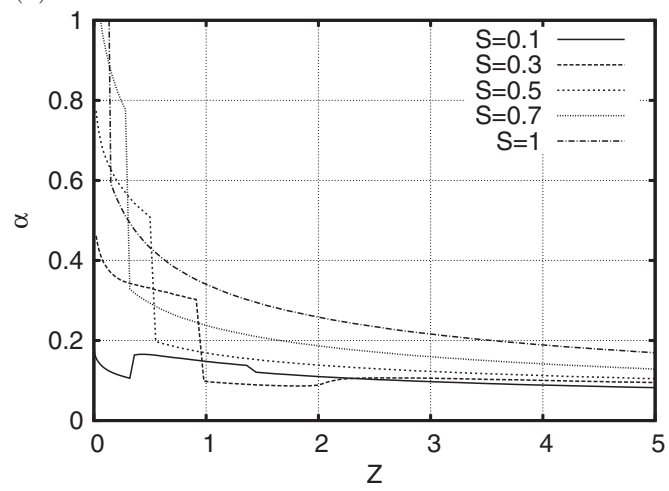

FIG. 10. (a) Critical curves and (b) corresponding wave-number plots for values of $S$ between 0.1 and 1 .

in $Z$. Note the sharp cusp in the critical curve for $S=6$, apparent in Fig. 12, which is a consequence of a jump between different local maxima of $\omega_{i}(\alpha)$ for $m=1$.

Figure 11(a) shows that the critical Re continues to be a decreasing function of $S$ for fixed $Z$ less than about 3. However, as is apparent from Fig. 12, this is no longer the case at larger $Z$ when $S$ exceeds a certain threshold value (which we found to be about 2.5). At such values of $S$, no general statement, covering all values of $Z$, concerning the effects of increasing rotation on flow stability can be made.

Figure 13(a) shows critical curves for $5<Z<100000$ and illustrates the statement, made earlier, that the critical Reynolds number tends to 0 as $Z \rightarrow \infty$. Figure 13(b) shows the same results, but it is $Z^{1 / 2} \operatorname{Re}$, rather than $\operatorname{Re}$, which is plotted as a function of $Z$. It appears that the curves asymptote to straight lines at large $Z$, corresponding to the asymptotic expansion $\operatorname{Re} \sim Z^{-1 / 2}(A \ln Z+B)$ for the critical Reynolds number as $Z \rightarrow \infty$. It is interesting to note that the coefficient $A$ (corresponding to the slope of the asymptote in the figure) appears to have the same value (close to 18) for different $S$. On the other hand, $B$ is a decreasing function of $S$, so increasing rotation destabilizes the flow at large $Z$, as it does at small $Z$. Although asymptotic analysis of the large- $Z$ limit of the stability problem has not been attempted, the form of the expansion given above is a logical consequence of that of the asymptotic expansions of the basic-flow velocity profiles, which were derived in I (Sec. 5a and Appendix A). These expansions proceed in inverse powers of $\ln Z$, hence, the appearance
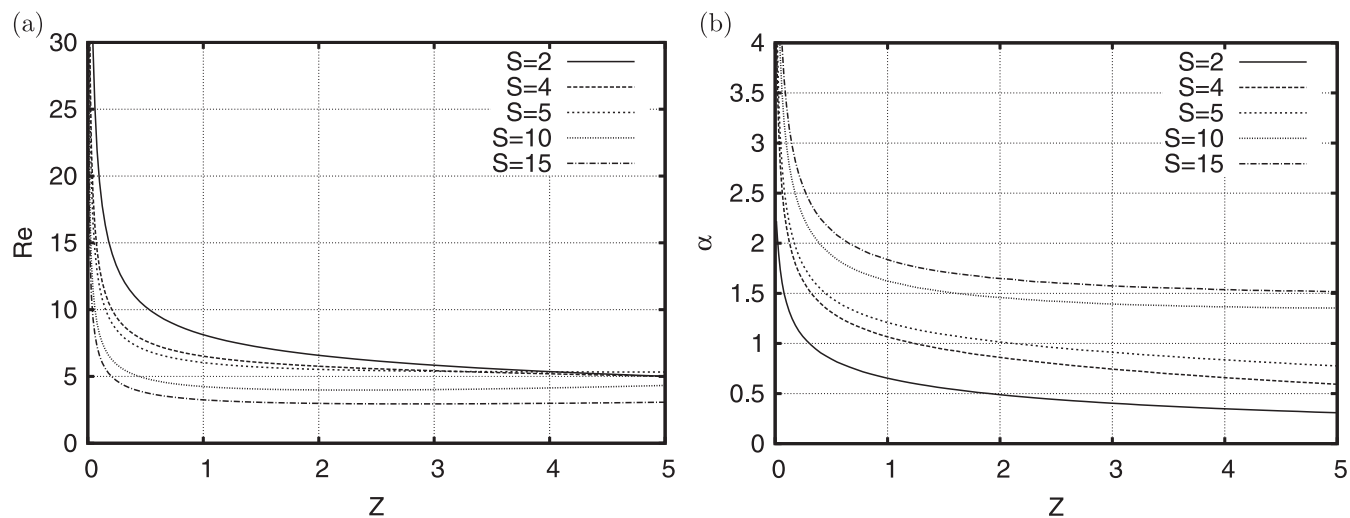

FIG. 11. (a) Critical curves and (b) corresponding wave-number plots for values of $S$ between 2 and 15 . 


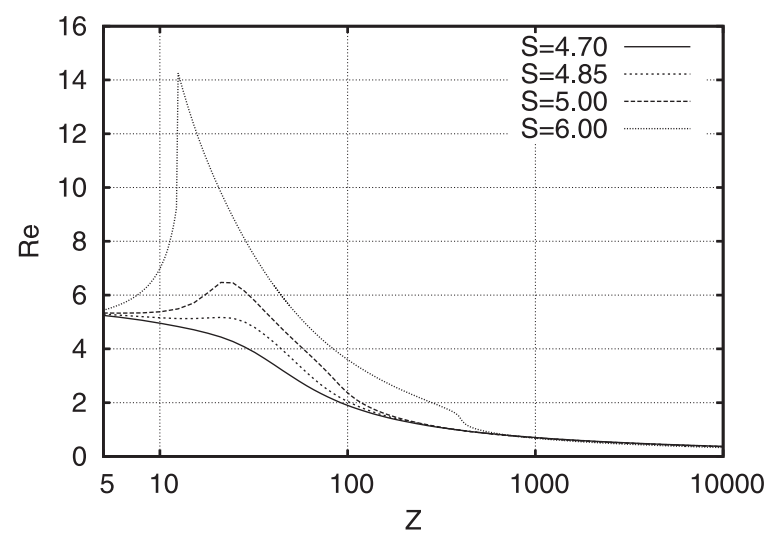

FIG. 12. Critical curves for $5<Z<10000$.

of $\ln Z$ in the expansion of the critical Reynolds number. The coefficients of the basic-flow expansions are functions of $r / Z^{1 / 2}$, leading to the factor of $Z^{-1 / 2}$.

Finally, some caveats concerning the results at large $S$. Figure 11(a) shows that the critical Reynolds number is rather low (of order 5) except at small $Z$. Such moderate values of Re raise questions concerning the basis of this work: both the boundary-layer equations used to compute the basic flow and the local stability equations for the perturbation are predicated on the assumption of large Re. Thus, accurate quantitative values of the critical Reynolds number should not be expected at large $S$. We might, nonetheless, hope that qualitative trends resemble the present results.

\section{Small- $S$ perturbation analysis}

As we have seen (recall Figs. 6 and 9), flow stability is significantly affected by small amounts of rotation. This suggests using the Taylor's series

$$
\omega_{i}=\omega_{i 0}+\frac{\partial \omega_{i}}{\partial S} S+\frac{1}{2} \frac{\partial^{2} \omega_{i}}{\partial S^{2}} S^{2}+\cdots
$$

to express the effects of rotation. Here, $\omega_{i 0}$ and the derivatives of $\omega_{i}$ denote $S=0$ values for given $m, Z, \operatorname{Re}$, and $\alpha$. As discussed earlier, small- $S$ stability is controlled by a single distinguished mode, having small $\omega_{i 0}$ and to which we specialize in this section (as noted earlier, other discrete eigenvalues are well below the real $\omega$ axis and hence unimportant).

(a)

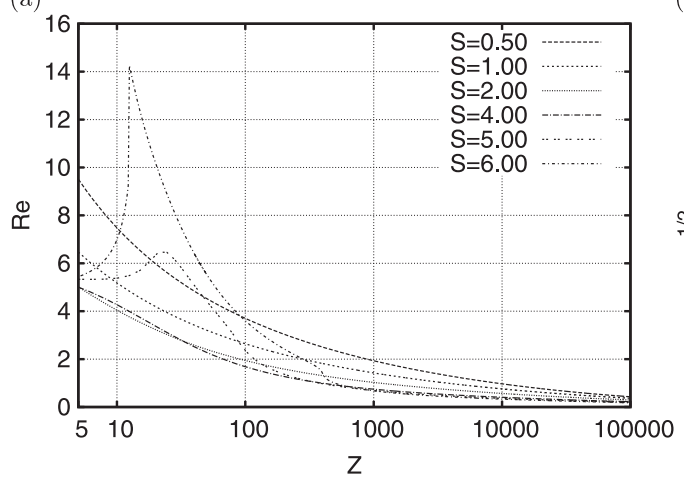

(b)

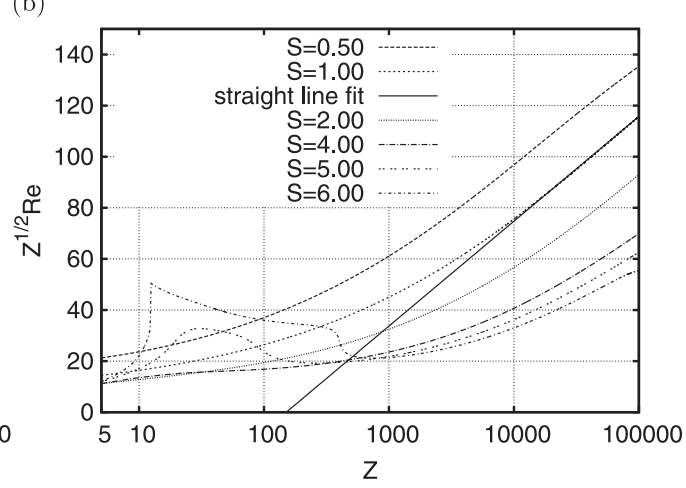

FIG. 13. Critical curves for $5<Z<100000$ : (a) Re, (b) $Z^{1 / 2} \operatorname{Re}$ as functions of $Z$. 


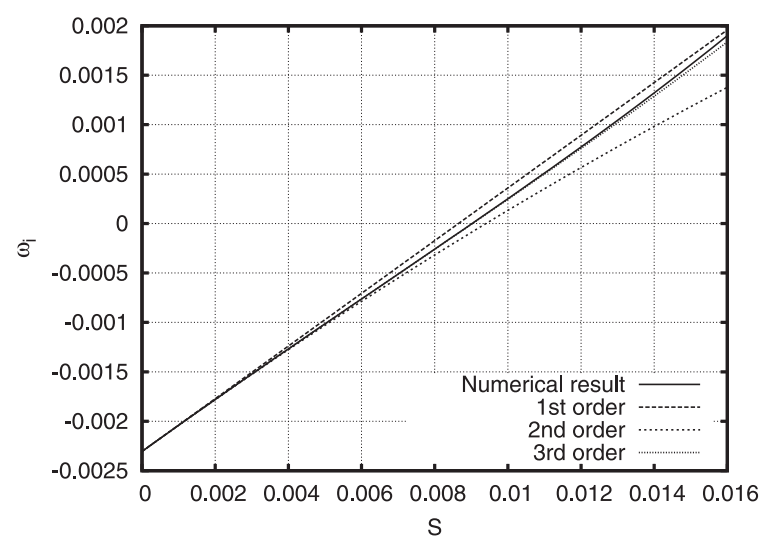

FIG. 14. Comparison of the growth rate of the most unstable mode obtained from the full numerical calculation and perturbation theory of different orders: $m=1, Z=1, \operatorname{Re}=2000, \alpha=0.1$.

Figure 14 shows a plot of $\omega_{i}(S)$ for particular values of $m, Z, \operatorname{Re}$, and $\alpha$. The full numerical results are compared with the Taylor's series truncated at different orders [the derivatives in Eq. (15) being determined using second-order, centered finite differencing). Second- and higher-order terms can be neglected to a first approximation. Note the small value of $\omega_{i 0}$, here negative, which is characteristic of the distinguished mode, and the order 1 value of $\partial \omega_{i} / \partial S$. It is the combination of these two ingredients which makes flow stability sensitive to small amounts of rotation. As noted earlier, increasing small $S$ destabilizes the flow, a result which is a consequence of positive $\partial \omega_{i} / \partial S$ for the distinguished mode when $S=0$.

\section{CONCLUSION}

In this paper, we have studied the local, linear stability of flow around a semi-infinite, rotating cylinder placed in an axial stream. Assuming large values of the Reynolds number, the basic flow is determined numerically using a boundary-layer approximation (as described in I). Large Reynolds number also justifies local stability analysis. The stability problem depends on three nondimensional physical parameters: the Reynolds number Re, the rotation rate $S$, and the axial location $Z$. Modal analysis introduces two additional parameters: namely, $\alpha$, the axial wave number, and $m$, which is an integer whose value identifies the azimuthal Fourier component of the mode. The local stability equations are solved numerically to obtain the complex frequency $\omega=\omega_{r}+i \omega_{i}$ of modes via solution of a matrix eigenvalue problem. The numerics were checked by studying the sensitivity of the results to variations of the numerical parameters of the problem and also by comparison with the few results available in the literature. The eigenspectrum consists of a discrete part and a numerical approximation (many closely spaced eigenvalues lying along a curve in the complex $\omega$ plane) of the continuous spectrum. The latter lies in $\omega_{i}<0$ and thus represents a decaying contribution which is unimportant from a stability point of view. Discrete eigenvalues in $\omega_{i}>0$ represent growing modes and are symptomatic of instability. Let $\omega_{i}^{\max }$ be the maximum growth rate, where only discrete eigenvalues contribute and the maximum is taken over $\alpha$ and $m$. If $\omega_{i}^{\max }>0$, the flow is unstable for the given values of $\operatorname{Re}, S$, and $Z$. This allows the determination of the stability boundary in physical-parameter space. The most important results of the paper consist of critical curves representing this boundary in the Z-Re plane for different values of $S$ (see Figs. 9-13).

An important conclusion of the paper is that flow stability is surprisingly sensitive to small amounts of rotation. This is apparent in Figs. 6 and 9(a) and is further quantified by the perturbation analysis of Sec. IV C. It is the consequence of a nearly neutral mode for $S=0$ which controls stability at small $S$. Even a small amount of rotation can produce a sufficient perturbation that $\omega$ crosses 
the real axis and flow stability or instability switches for the given values of Re and $Z$. Rotation is destabilizing for small $S$, i.e., the critical Reynolds number decreases for fixed $Z$ as $S$ is increased.

As is apparent from Fig. 9(a), in the absence of rotation the flow is stable beyond a certain value of $Z$ (close to 0.8 ) and also below a Re threshold (close to 1060). No such constraints on instability apply when rotation is added. As for $S=0$, the critical Reynolds number goes to infinity at small $Z$, but, for nonzero $S$, it approaches zero as $Z \rightarrow \infty$. Thus, as in the nonrotating case, the flow is stable at sufficiently small $Z$, but, with rotation, it is unstable at large enough downstream distances.

Between small and large $Z$ there is at least one boundary separating stable and unstable portions of the flow. The simplest case is when the critical Reynolds number is a monotonic decreasing function of $Z$. There is then a single stability boundary in $Z$, below which the flow is stable, and above which it is unstable. On the other hand, a nonmonotonic critical curve implies the possibility, dependent on Re, of more than one stability boundary in $Z$. We found that the critical curve was monotonic for $S$ between about 0.0045 and 5 .

As noted above, rotation is destabilizing at small $S$. This was found to persist up to about $S=2.5$, but beyond that there were cases for which the critical Reynolds number increases with increasing $S$ at fixed $Z$. Even above this threshold, the destabilizing trend continues for $Z$ below about 3 and at sufficiently large values of $Z$. Destabilization by rotation can thus be considered as the norm.

Finally, the limit of large $Z$ was also examined. We found that the critical Reynolds number has the asymptotic expansion $\operatorname{Re} \sim Z^{-1 / 2}(A \ln Z+B)$, where the factor $A$ has the same value for all $S$ and $B$ is a decreasing function of $S$. The expansion is consistent with the earlier statement that critical Re goes to zero as $Z \rightarrow \infty$, while decreasing $B$ means that rotation destabilizes the flow at large $Z$, as stated above.

\section{ACKNOWLEDGMENTS}

The authors are grateful to the French Ministry of Education and Research and the Indo-French Centre for the Promotion of Advanced Research-CEFIPRA for financial support.

[1] H. L. Reed and W. S. Saric, Stability of three-dimensional boundary layers, Annu. Rev. Fluid Mech. 21, 235 (1989).

[2] W. S. Saric, H. L. Reed, and E. B. White, Stability and transition of three-dimensional boundary layers, Annu. Rev. Fluid Mech. 35, 413 (2003).

[3] R. J. Lingwood, Absolute instability of the boundary layer on a rotating disk, J. Fluid Mech. 299, 17 (1995).

[4] B. Pier, Primary crossflow vortices, secondary absolute instabilities and their control in the rotating-disk boundary layer, J. Eng. Math. 57, 237 (2007).

[5] S. J. Garrett and N. Peake, The absolute instability of the boundary layer on a rotating cone, Eur. J. Mech. B: Fluids 26, 344 (2007).

[6] S. J. Garrett, Z. Hussain, and S. O. Stephen, Boundary-layer transition on broad cones rotating in an imposed axial flow, AIAA J. 48, 1184 (2010).

[7] B. Pier, Periodic and quasiperiodic vortex shedding in the wake of a rotating sphere, J. Fluids Struct. 41, 43 (2013).

[8] S. Derebail Muralidhar, B. Pier, J. F. Scott, and R. Govindarajan, Flow around a rotating, semi-infinite cylinder in an axial stream, Proc. R. Soc. London, Ser. A 472, 20150850 (2016).

[9] W. Tollmien, Über die Entstehung der Turbulenz. 1. Mitteilung, Nachr. Ges. Wiss. Goettingen, Math. Phys. Kl. 1929, 21 (1928).

[10] R. Jordinson, The flat plate boundary layer. Part 1. Numerical integration of the Orr-Sommerfeld equation, J. Fluid Mech. 43, 801 (1970). 
[11] L. M. Mack, A numerical study of the temporal eigenvalue spectrum of the Blasius boundary layer, J. Fluid Mech. 73, 497 (1976).

[12] O. R. Tutty, W. G. Price, and A. T. Parsons, Boundary layer flow on a long thin cylinder, Phys. Fluids 14, 628 (2002).

[13] N. Vinod and R. Govindarajan, Secondary instabilities in incompressible axisymmetric boundary layers: Effect of transverse curvature, J. Fluids Eng. 134, 024503 (2012).

[14] K. H. Kao and C. Y. Chow, Stability of the boundary layer on a spinning semi-infinite circular cylinder, J. Spacecr. Rockets 28, 284 (1991).

[15] M. A. Herrada, C. Del Pino, and R. Fernandez-Feria, Stability of the boundary layer flow on a long thin rotating cylinder, Phys. Fluids 20, 034105 (2008).

[16] J. T. Kegelman, R. C. Nelson, and T. J. Mueller, The boundary layer on an axisymmetric body with and without spin, AIAA J. 21, 1485 (1983).

[17] M. G. Hall, Vortex breakdown, Annu. Rev. Fluid Mech. 4, 195 (1972).

[18] C. Leclercq, Instabilités convectives et absolues dans l'écoulement de Taylor-Couette-Poiseuille excentrique, Ph.D. thesis, École centrale de Lyon, 2013.

[19] P. J. Schmid and D. S. Henningson, Stability and Transition in Shear Flows, Applied Mathematical Sciences (Springer, New York, 2001).

[20] L. N. Howard and A. S. Gupta, On the hydrodynamic and hydromagnetic stability of swirling flows, J. Fluid Mech. 14, 463 (1962).

[21] T. J. Pedley, On the instability of viscous flow in a rapidly rotating pipe, J. Fluid Mech. 35, 97 (1969). 